\title{
Shimada Classification System
}

National Cancer Institute

\section{Source}

National Cancer Institute. Shimada Classification System. NCI Thesaurus. Code C85413.

A classification system defining a neuroblastoma as having a favorable or unfavorable prognosis, based on histopathologic criteria. 